\title{
DIMENSIONES DE CULTURA NACIONAL QUE AFECTARÍAN LA PRÁCTICA DEL LIDERAZGO DE SERVICIO: APLICACIÓN AL CASO ECUATORIANO
}

\section{DIMENSIONS OF NATIONAL CULTURE THAT WOULD AFFECT THE PRACTICE OF SERVANT LEADERSHIP: APPLICATION TO THE ECUADORIAN} CASE

CHRISTIAN BENJAMÍN CABEZAS GUERRA ${ }^{1}$ DAMIÁN ALEXANDER ORTIZ HEREDIA ${ }^{2}$

Recibido: 15 de mayo de 2017 Aceptado: 29 de agosto de 2017

\footnotetext{
${ }^{1}$ Pontificia Universidad Católica del Ecuador, Facultad de Psicología, Quito, Ecuador (chcabezas@puce.edu.ec).

${ }^{2}$ Pontificia Universidad Católica del Ecuador, Facultad de Psicología, Quito, Ecuador (dortiz806@puce.edu.ec).
} 



\title{
DIMENSIONES DE CULTURA NACIONAL QUE AFECTARÍAN LA PRÁCTICA DEL LIDERAZGO DE SERVICIO: APLICACIÓN AL CASO ECUATORIANO
}

\section{NATIONAL CULTURE DIMENSIONS THAT WOULD AFFECT THE PRACTICE OF SERVANT LEADERSHIP: APPLICATION TO THE ECUADORIAN CASE}

\author{
Christian Benjamín Cabezas Guerra y Damián Alexander Ortiz Heredia
}

PALABRAS CLAVE: Liderazgo de Servicio, Cultura Nacional, Dimensiones Culturales, Ecuador.

KEY WORDS: Servant-Leadership, National Culture, Cultural Dimensions, Ecuador.

\section{RESUMEN}

El objetivo de este trabajo es presentar el tema del Liderazgo de Servicio (Servant Leadership), sus principales características, sus efectos en las organizaciones, las variables de cultura nacional que afectarían su aplicabilidad, y el desarrollo de un análisis de su posible aplicación específica al contexto ecuatoriano.
Este trabajo se ha realizado mediante la recopilación de estudios teóricos y empíricos sobre la aplicación de este estilo de liderazgo en diferentes contextos y la variabilidad del funcionamiento del mismo dependiendo del contraste de contextos culturales nacionales. Este tipo de liderazgo ético basado en el inte- 
rés por desarrollar a otros, podría ser de aplicación global, aunque su aceptación y funcionamiento podría variar levemen- te dependiendo de las dimensiones y características culturales de cada nación específica.

\section{ABSTRACT}

This article reviews the topic of Servant Leadership, its main conceptualization, its effects on organizations, the variables of national culture that would affect its applicability, and the application of an analysis of these variables to the Ecuadorian context. This work was performed through the collection of theoretical and empirical studies on the application of this leadership style in different contexts and the variability of its functioning depending on the difference of national cultural contexts. This type of ethical leadership based on the interest of developing others, could be of global application although its level of success could vary slightly depending on the dimensions and cultural characteristics of each specific nation. The study's conclusions and suggestions for future research related to this topic are presented at the end of this article.

\section{INTRODUCCIÓN}

La teoría de Liderazgo de Servicio (Greenleaf, 1977) se considera como una perspectiva diferente del liderazgo puesto que reconoce la importancia de la relación entre los líderes y los seguidores de una manera única. Greenleaf (1977, pág. 13) afirmó que "la diferencia se manifiesta en el cuidado que el servidor tiene de asegurarse que las necesidades primordiales de los otros estén siendo satisfechas". Greenleaf también sostuvo que "un gran líder debe ser visto primero como servidor, y ese mismo hecho es la clave de su grandeza" (1977, pág. 21). Esta perspectiva contrasta con otras formas de liderazgo que se enfocan en el logro de objetivos organizacionales como la prioridad de la práctica del liderazgo, y miran al desarrollo de otros como medios para conseguir esos objetivos (Smith, Montagno y Kuzmenko, 2004).

Con su propuesta de que un líder debe ser un servidor primero, esta teoría rompió diferentes paradigmas existentes hasta ese momento en lo 
que respecta la naturaleza del liderazgo. Este tipo de liderazgo es considerado único puesto que la descripción de sus características comienza con un análisis del sistema de creencias y valores del líder, y su motivación para querer liderar (Smith et al., 2004).

Varios estudios empíricos recientes han mostrado evidencias de los beneficios de adoptar este liderazgo dentro de diversas organizaciones. Por ejemplo, en una extensa revisión de la bibliografía existente sobre el Liderazgo de Servicio, Van Dierendonck (2010) presentó un resumen de diferentes hallazgos empíricos que demuestran los beneficios de este estilo de liderazgo. De acuerdo con Van Dierendonck (2010, pág. 21), existe abundante evidencia empírica de estudios transversales que demuestra que los líderes de servicio "hacen que los empleados estén más satisfechos, comprometidos, y muestren mejor desempeño" en comparación con otros tipos de líderes. Según este mismo autor, los meta-análisis sobre el liderazgo de servicio demuestran que existen tres cambios específicos en los empleados que perciben este tipo de liderazgo en sus organizaciones: crecimiento personal en términos de autorrealización, cambio en lo que respecta a mejora de actitudes hacia el trabajo y transformación en términos de trabajo en equipo y colaboración con otros.
En otro estudio desarrollado con el objetivo de evaluar de una manera general el verdadero impacto del Liderazgo de Servicio en los contextos organizacionales, Parris y Peachey (2013) mediante una revisión sistemática de literatura (RSL) analizaron los resultados de 39 investigaciones en contextos organizacionales en los que se aplicó este tipo de liderazgo. Los resultados mostraron que la teoría de liderazgo de servicio es viable y ayuda a mejorar el bienestar de los empleados en estos contextos. Una de las principales conclusiones de ese trabajo fue que "la teoría del Liderazgo de Servicio es aplicable en una variedad de culturas, contextos, y ambientes organizacionales" (Parris y Peachey, 2013, pág. 388).

Aunque varios estudios han analizado el impacto positivo que tendría el Liderazgo de Servicio en las instituciones, no se ha profundizado lo suficiente en las variables o factores que afectarían o influenciarían esa aplicabilidad en diferentes contextos. Se han realizado algunos estudios empíricos que han encontrado que la práctica del Liderazgo de Servicio podría estar afectada por dimensiones culturales nacionales (Carroll y Patterson, 2014; Hale y Fields, 2007; Han, Kakabadse y Kakabadse, 2010; Hannay, 2009; Top, Öge, Atan, y Gümüs, 2015), variables organizacionales (Harwiki, 2016; Koesmono, 2014; Song, Park y 
Kang, 2015; Top et al., 2015), variables individuales (Barbuto, Gottfredson y Searle, 2014; Beazley y Gemmill, 2006; Sun, 2013; Tulasi Das y Anand Reddy, 2013; Van Dierendonck, 2011; Verdorfer, 2016; Washintong, Sutton y Field, 2006) y variables demográficas (Parris y Peachey, 2013).

En lo que respecta específicamente a las variables de cultura nacional, algunos autores han sugerido que el Liderazgo de Servicio podría ser más fácilmente aplicado en culturas con algunas características específicas. De acuerdo con Rubio-Sánchez, Bosco y Melchar (2013), la diferencia de valores culturales entre países produciría que el estilo de Liderazgo de Servicio pueda tener mejores resultados en culturas en las cuales se apoyan valores similares a los que este liderazgo promulga. Estos mismos autores examinaron la congruencia de los valores que promueve este liderazgo con los valores nacionales de diferentes países identificados en la encuesta mundial de valores (World Values Association, World Values Survey, 2013). Los resultados de esta investigación sugirieron que este tipo de liderazgo podría encajar mejor en algunos países que en otros. De acuerdo con Rubio-Sánchez, et al., (2013), el Liderazgo de Servicio sería de mejor aplicabilidad en Estados
Unidos, Bangladesh, Suecia, Canadá y Tanzania, mientras que sería de menor aplicabilidad en Rusia, Lituania, Letonia, Bielorrusia, Ucrania, Estonia y Turquía. Los autores concluyeron que este liderazgo encajaría de mejor manera en culturas en las cuales se valora la iniciativa, libertad de decisión, confianza y autonomía.

Existen otros estudios no empíricos que han sugerido que el Liderazgo de Servicio sería de mejor aplicabilidad en culturas nacionales con niveles específicos en sus dimensiones. Por ejemplo, Hannay (2009), analizando las características de las dimensiones culturales propuestas por Hofstede (1980), sugirió que este liderazgo sería de mejor aplicabilidad en culturas que muestran baja distancia de poder, bajo o moderado individualismo, baja o moderada masculinidad, bajo nivel de evitación de la incertidumbre y una alta o moderada orientación al largo plazo.

El presente trabajo trata de explorar mediante una revisión de trabajos teóricos y empíricos la influencia que las dimensiones de cultura nacional podrían tener sobre la aplicabilidad del liderazgo de servicio en general y, posteriormente, de una manera singular en el contexto ecuatoriano con sus niveles específicos de dimensiones culturales. 


\section{PREGUNTAS DE INVESTIGACIÓN (OBJETIVOS GENERAL Y ESPECÍFICOS)}

Este trabajo se plantea la pregunta general: ¿existe una influencia en la aplicación del liderazgo de servicio dependiendo de los niveles de las dimensiones culturales nacionales?

Las preguntas específicas que este proyecto investigará son:
- ¿Existe una mejor posibilidad de aplicación del liderazgo de servicio dependiendo de niveles específicos de dimensiones culturales?

- ¿ ¿Cómo sería la aplicación del liderazgo de servicio en el caso ecuatoriano de acuerdo a sus niveles de dimensiones culturales?

\section{REFERENTES TEÓRICOS}

\section{El Liderazgo de Servicio}

Robert Greenleaf (1977) se refirió al Liderazgo de Servicio (Servant-Leadership) como la nueva forma de liderazgo necesaria para sanar a las sociedades. Este autor enunció: “El líder de servicio es primero servidor ... comienza con el sentimiento natural de que uno quiere servir primero ... esa persona es muy distinta de la que es líder primero, tal vez debido a la necesidad de este último de apaciguar una unidad de poder o de adquirir posesiones materiales"(pág. 27). En la actualidad, el mundo parece necesitar este tipo de líder, que no se preocupa únicamente por hacer de su nación u organización un imperio que domine económicamente y políticamente a otros, sino un individuo corresponsable del desarrollo global, considerando que la explotación huma- na y ambiental eventualmente afectará sus propios intereses.

Greenleaf (1977, pág. 27) subrayó la diferencia entre una persona que elige ser un líder primero y una persona (líder de servicio) que elige ser un servidor primero. Los líderes de servicio se aseguran de que las necesidades más prioritarias de otras personas están siendo atendidas. Mencionó que la mejor prueba para identificar esta cualidad es preguntar: "¿Los individuos que son atendidos por un líder de servicio crecen como personas? ¿Se sienten más sanos, más sabios, más libres, más autónomos, más propensos también a servir?"...

Estas aseveraciones del pionero del Liderazgo de Servicio son importantes puesto que nos dan un marco básico de referencia para analizar nuestro papel 
en las instituciones donde actualmente trabajamos y la influencia de estas en nuestras sociedades. ¿Estamos en nuestras instituciones ayudando a otros a crecer? ¿Están nuestras instituciones practicando el liderazgo de servicio, haciendo a sus trabajadores y a otras partes involucradas personas más sanas, más sabias, más libres y más autónomas?

Con su definición de este tipo de liderazgo, Greenleaf propuso una revolución silenciosa en la forma en que vemos y practicamos el liderazgo. Actualmente, el concepto de Liderazgo de Servicio es cada vez más visto como una forma de liderazgo atractiva y que produce claros beneficios. Muchas empresas y organizaciones de distinto tipo están actualmente cambiando los modelos autocráticos y jerárquicos más tradicionales de liderazgo y se están volcando hacia el Liderazgo de Servicio como una forma de desarrollo de los demás y de autodesarrollo. De acuerdo a Spears (2005), este tipo de liderazgo busca involucrar a otros en la toma de decisiones, y se basa fuertemente en el comportamiento ético y de cuidado, y mejora el crecimiento de los trabajadores mientras mejora el cuidado y la calidad de la vida organizacional.

\section{Características del Liderazgo de Servicio}

Varios autores han recogido las primeras definiciones de Greenleaf y su caracterización del Liderazgo de Servicio para establecer diferentes análisis de su naturaleza y características. Por ejemplo, en un estudio de literatura sobre el Liderazgo de Servicio, Russell (2001) estableció que el mismo, al estar enfocado en el bienestar de los demás, tiene como elementos precursores principales a los valores. También sugirió que los valores son las variables independientes que predicen e impulsan el comportamiento de los líderes de servicio e identificó como los valores claves del liderazgo de servicio a la humildad, el respeto por los demás, la honestidad, la integridad, la equidad, y el amor. Consecuentemente, cuando se ha intentado asignar características a este tipo de liderazgo, por su naturaleza única, la descripción de las mismas comienza con un análisis del sistema de creencias y valores del líder, y su motivación para liderar.

Diferentes autores han identificado en sus trabajos a las características más importantes de este tipo de liderazgo. Uno de los trabajos más reconocidos con este fin fue realizado por Russell y Stone (2002), quienes mediante un análisis conceptual, señalaron las principales características de este tipo de liderazgo: escucha activa, empatía, sanación, atención, persuasión, conceptualización, previsión, administración, compromiso con el crecimiento de la gente y generación de comunidad. De acuerdo a Spears (co- 
municación personal, 2012), estas diez características del Liderazgo de Servicio no son de ninguna manera exhaustivas. Sin embargo, sirven para comunicar el poder y la promesa que ofrece este concepto a aquellos que están abiertos a su invitación y desafío. Spears (1998) también sugirió que las características del Liderazgo de Servicio a menudo ocurren naturalmente en muchos individuos, y pueden ser mejoradas a través del aprendizaje y la práctica.

El primer estudio empírico con el objeto de caracterizar y medir el Liderazgo de Servicio fue realizado por Laub (1999), que encuestó a 847 personas de 41 organizaciones y desarrolló un instrumento de 43 ítems con el cual midió seis dimensiones de este liderazgo: desarrollo de personas, liderazgo compartido, autenticidad, valoración de personas y construcción de la comunidad. Algunos trabajos posteriores con este mismo objetivo incluyen los estudios de Page y Wong (2000), Dennis y Bocarnea (2005), Barbuto y Wheeler (2006), Liden, Wayne, Zhao y Henderson (2008), van Dierendonck (2011), Van Dierendonck y Nuijten (2011) y Reed, Vidaver-Cohen y ColweII (2011). Uno de los últimos trabajos que ha servido mucho para posteriores investigaciones, incluyendo la presente, fue realizado por Mittal y Dorfman (2012) quienes con base a diferentes operacionalizaciones del liderazgo de servicio en estudios empíricos propusieron que el mismo está compuesto por las siguientes dimensiones: egalitarianismo, integridad moral, empoderamiento y desarrollo de otros, empatía y humildad.

\section{Cultura Nacional y Liderazgo de Servicio}

Desde que Greenleaf (1970) formuló el concepto de liderazgo de servicio, numerosos autores se han interesado en identificar qué variables podrían facilitar o dificultar su práctica y aplicabilidad. Una de las principales variables que se ha estudiado y que tendría un efecto sobre la práctica y el éxito en el funcionamiento del Liderazgo de Servicio es la cultura nacional y sus dimensiones.

\section{Teorías de dimensiones culturales}

La cultura es un concepto que se ha abordado por diferentes disciplinas con diversidad de métodos, esto ha derivado en que se la comprenda bajo una multiplicidad de definiciones (Kroeber y Kluckhon, 1952). Según Molero (2002, pág. 53), las conceptualizaciones sobre cultura suelen coincidir en que "la cultura está constituida por un conjunto de creencias, actitudes, valores y prácticas compartidas por un grupo de personas con una historia común e insertas en una estructura social determinada".

Con el fin de analizar la cultura se han propuesto diversas perspectivas 
para entenderla. Machado y Carvalho (2008) identificaron dos grandes grupos en estas propuestas teóricas: 1) aquellas que se prestan mayoritariamente para métodos de investigación cualitativa (por ejemplo, Triandis -1994-; Trompenaars y Hampden-Turner -1997-) y 2) aquellas orientadas a su aplicación en métodos cuantitativos (por ejemplo, Hofstede, 1980; House, Hanges, Javidan, Dorman y Gupta, 2004), que permiten medir elementos de la cultura, relacionar dichos elementos con variables de interés, así como comparar diferentes grupos humanos.

Como señalan Chanchani y Theivananthampillai (2009, pág. 3) "la literatura está repleta con tipologías culturales", entre las cuales podemos enlistar las propuestas de Cattell (1949); Sawyer (1967); Rummell (1972); Inkeles y Levinson (1969). En virtud de la amplitud de las propuestas, se especificarán y aplicarán en este trabajo aquellas que han tenido mayor impacto en estudios empíricos (Shi y Wang, 2011): las propuestas de Hosftede (1980) y el proyecto GLOBE (Global Leadership and Organizational Behavior Effectivenes) de House et al. (2004).

La Teoría de las 5 dimensiones culturales de Hofstede: El proyecto de Hofstede (1980) presentó uno de los análisis pioneros y más amplios de la cultura con rango internacional (66 países).
El análisis inicial de la cultura de Hofstede reconoció únicamente cuatro dimensiones. Posteriormente, Hofstede y Bond (1988) añadieron al estudio original una quinta dimensión denominada "orientación a largo vs. corto plazo". Las 5 dimensiones culturales del modelo de Hofstede: Individualismo vs. Colectivismo; Alta vs. baja distancia del poder; Alta vs. baja evitación de la incertidumbre; Masculinidad vs. Femineidad; y Orientación a largo vs. corto plazo

\section{El programa de investigación}

GLOBE: Este proyecto estudió mediante diversos métodos de medición nueve dimensiones culturales en 62 culturas societales (House et al., 2004) con el fin de "predecir el impacto de variables específicas culturales en el liderazgo y en procesos organizacionales, así como en la efectividad de estos procesos" (House, Javidan, Hanges y Dorfman, 2002, pág. 4). House et al. prefirieron el uso del término "culturas societales" en contraposición a "naciones", con la finalidad de no pasar por alto la complejidad cultural de algunas naciones (por ejemplo, Alemania se dividió en antigua Alemania Oriental y antigua Alemania Occidental).

Las nueve dimensiones estudiadas fueron medidas tanto en una muestra de la sociedad en general como de una organización específica. Además, se diferenció entre "prácticas" (cómo son los hechos con respecto a una dimensión 
cultural) y "valores" (cómo piensan las personas que deberían ser los hechos). Las dimensiones estudiadas por House et al. (2004) son: Evitación de la incertidumbre; Distancia de poder; Colectivis- mo I (institucional); Colectivismo || (de grupo); Igualdad de género; Asertividad; Orientación al futuro; Orientación al desempeño; y Orientación humana

\section{DIMENSIONES CULTURALES DEL ECUADOR DE ACUERDO A HOFSTEDE Y AL ESTUDIO GLOBE}

De acuerdo con las evaluaciones realizadas a partir de la teoría de Hofstede (2017), y al estudio GLOBE (2004), la cultura ecuatoriana tiene las siguientes características principales en sus dimensiones:

De acuerdo a Hofstede (2017), la puntuación más alta de la dimensión cultural del Ecuador es "Distancia del Poder"(PDI) con una puntuación de 78. Una alta puntuación en esta dimensión indica un alto nivel de desigualdad de poder y riqueza dentro de la sociedad. Otra dimensión cultural manifiesta del Ecuador es la "Evitación de la incertidumbre" con un puntaje de 67, lo que indica el bajo nivel de tolerancia de la sociedad por la incertidumbre. En un esfuerzo por minimizar o reducir este nivel de incertidumbre, se adoptan e implementan reglas estrictas, leyes, políticas y reglamentos. Hofstede sugiere que el objetivo final de poblaciones con niveles altos en esta dimensión es controlar todo para eliminar o evitar lo inesperado.
Ecuador tiene uno de los puntajes más bajos en la dimensión de "Individualismo" (8), en comparación con otros países latinoamericanos (promedio 21). Esta puntuación indica que la sociedad es eminentemente "colectivista". En las culturas colectivistas, la familia y la comunidad son más importantes que las regulaciones sociales. La sociedad fomenta relaciones fuertes en las cuales cada uno asume la responsabilidad de los compañeros de su grupo. Los estudios de Hofstede no han reportado en los últimos años datos sobre los niveles de las dimensiones "Orientación a largo plazo" e "Indulgencia" para la cultura ecuatoriana. Los indicadores de dimensiones culturales de la cultura ecuatoriana anteriormente mencionados coinciden con las medidas desarrolladas por el Programa de Investigación GLOBE. En este estudio, Ecuador, al igual que otras culturas latinoamericanas, se caracteriza por el colectivismo grupal y un nivel significativo de evitación de la incertidum- 
bre. El estudio GLOBE, además, señala que Ecuador, junto con otras culturas similares de la región, se caracteriza por una orientación a corto plazo, (Northouse, 2004, pág. 331).

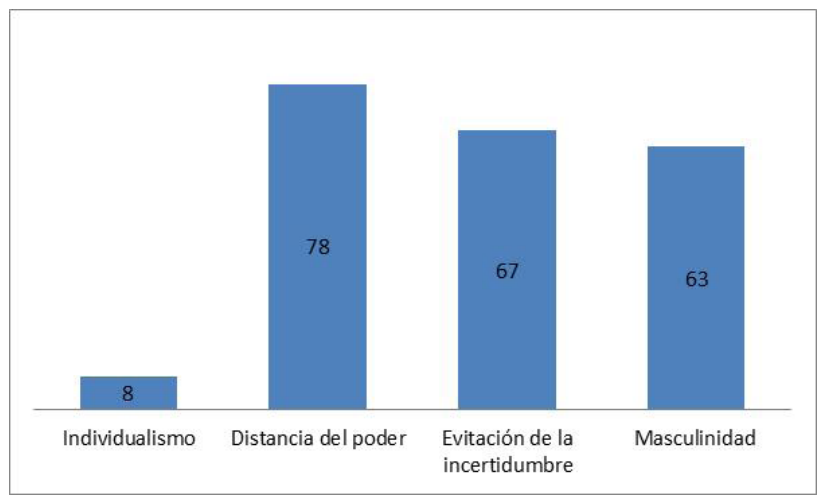

Figura 1: Niveles de las dimensiones culturales del Ecuador. Fuente: Hofstede, 2016.

\section{APLICACIÓN DEL LIDERAZGO DE SERVICIO DEPENDIENDO DE LAS DIMENSIONES DE CULTURA NACIONAL}

En las últimas décadas ha existido mucho interés en estudiar la aplicabilidad del liderazgo de servicio dependiendo de la cultura nacional. Referente al estudio de este último constructo se han utilizado las tipologías culturales de Hofstede (1980) y el estudio GLOBE (House et al., 2004). Se han aplicado algunos métodos para examinar la relación entre las dimensiones culturales propuestas por esas teorías con el Liderazgo de Servicio. Entre algunos de estos estudios tenemos los de Carroll y Patterson (2014); Hale y Fields (2007); Han, Kakabadse y Kakabadse, (2010); Top, Öge, Atan y Gümüs (2015). Los resultados de estos estudios generalmente han apuntado a que, aunque el liderazgo de servicio parece ser aplicable en diferentes culturas, algunos aspectos tienen más 
importancia que otros en culturas específicas de acuerdo a sus dimensiones y características culturales.

Los estudios iniciales sobre la aplicación de este tipo de liderazgo en diferentes contextos culturales fueron desarrollados primordialmente mediante un análisis conceptual en el que se infería la aplicabilidad del Liderazgo de Servicio dependiendo de una congruencia teórica de sus características con la descripción de las dimensiones culturales específicas de los modelos como el de Hofstede y el estudio GLOBE. Por ejemplo, en el estudio GLOBE (Javidan, House y Dorfman, 2004) se concluyó que varias características de liderazgo son culturalmente contingentes. Son deseables en algunas culturas, pero indeseables en otras. En este estudio se sugirió, por ejemplo, que un liderazgo orientado hacia la humanidad parece ser muy deseable en las sociedades del sur de Asia, mientras que en Europa nórdica no se consideraría tan importante.

Además, en algunas culturas, como los Estados Unidos, el concepto de liderazgo se considera idealizado y los líderes tienen privilegios y estatus excepcionales, mientras que en Holanda y Suiza se rechazaría esa idealización y se enfocarían más en el trabajo hacia el beneficio común. En estas naciones a los líderes no se les otorgaría ningún estatus o privilegio especial. Sobre este mismo tema, Choi y Yoon (2005) sugirieron que algunos comportamientos específicos se percibirían como conductas de liderazgo eficaces en culturas con niveles opuestos en dimensiones específicas. Por ejemplo, los comportamientos de auto-sacrificio se percibirían como eficaces en culturas con niveles de distancia de poder altas y bajas, así como en culturas individualistas y colectivistas.

Un buen número de estudios empíricos sobre la aplicación del Liderazgo de Servicio en diferentes contextos culturales se han desarrollado con el objetivo de comparar el funcionamiento del mismo entre dos culturas nacionales específicas. Así, Hale y Fields (2007) exploraron la diferencia de la práctica y percepción del Liderazgo de Servicio en Ghana y los EE.UU, y encontraron que los participantes de Ghana experimentaban comportamientos de este tipo de liderazgo significativamente menos frecuentemente que sus homólogos estadounidenses. Hale y Fields sugirieron que los mayores niveles de distancia de poder y colectivismo en la cultura ghanesas influenciaban en estos resultados. Ellos argumentaron que, dado que el Liderazgo de Servicio requiere que los líderes de alto rango demuestren humildad, los líderes de Ghana podrían haber encontrado que era incompatible con las normas de conducta que aceptan desigualdades (alta distancia de poder). 
En un estudio similar, Pekerti y Sendjaya (2010), al comparar la valoración del Liderazgo de Servicio entre Australia e Indonesia encontraron que los individuos de ambas culturas valoraban al concepto de este estilo de liderazgo de una manera similar, pero tenían diferencias culturales específicas sobre lo que se considera importante entre las diferentes dimensiones que los conforman. Este hallazgo evidencia que este tipo de liderazgo puede ser apreciado de manera similar en culturas con dimensiones diferentes en distancia de poder (alta/ baja) e individualismo (vs. colectivismo). No obstante, se apreciarían de manera diferente elementos específicos de este tipo de liderazgo.

Además de los estudios de comparación de la aplicación del Liderazgo de Servicio entre dos culturas específicas, en los últimos años se han realizado otras investigaciones con el objetivo de evaluar la aplicación del Liderazgo de Servicio en diferentes regiones o a nivel mundial dependiendo de diferencias culturales. En ese sentido, Rubio-Sánchez, Bosco y Melchar, (2013) examinaron las variables que componen la Encuesta de Valores Mundiales (World Values Survey) y seleccionaron aquellas que se percibían como directamente relacionadas con el liderazgo de servicio en el lugar de trabajo. Se encontró que las variables eran valoradas significati- vamente de una manera diferente entre las culturas nacionales. Por lo tanto, las percepciones de las características que componen el modelo de Liderazgo de Servicio difieren significativamente de una nación a otra.

En este análisis se pudo observar que hay naciones cuyos valores culturales son más congruentes con el modelo de Liderazgo de Servicio. Por ejemplo, de acuerdo a Rubio-Sánchez, Bosco y Melchar (2013), en los Estados Unidos los valores culturales nacionales, se beneficiarían de un estilo de liderazgo que incorpora las características de un líder de servicio, tales como: la importancia del trabajo en la vida, tener la oportunidad de usar la iniciativa y la libertad en la toma de decisiones en un trabajo. Los empleados en Bangladesh, Suecia y Canadá también encontraban estas características importantes para sus relaciones de liderazgo. Por otra parte, se encontraron algunos países en los que los valores nacionales no eran completamente congruentes con el Liderazgo de Servicio. Los rusos, seguidos de cerca por los Letones y Lituanos, obtuvieron una puntuación baja en los valores congruentes con el Liderazgo de Servicio,

En un estudio similar, Mittal y Dorfman (2012), basándose en los resultados del estudio GLOBE, analizaron el grado en que se aceptaban como características de un liderazgo eficaz a 
cinco aspectos del liderazgo de servicio identificados en estudios empíricos (Egalitarianismo, Integridad Moral, Empoderamiento, Empatía y Humildad). Este análisis lo hicieron comparando la aceptación de este estilo de liderazgo en grupos conformados por culturas diferenciadas geográficamente. Basándose en el cálculo del análisis de varianza (ANOVA), estos autores encontraron que cuatro de las cinco dimensiones del Liderazgo de Servicio son percibidas como de diferente importancia por los diferentes grupos de naciones. Estas dimensiones son Egalitarianismo, Empoderamiento, Empatía y Humildad. La dimensión de Integridad Moral no tuvo diferencia significativa en su valoración de importancia entre los grupos de naciones, lo que nos permite pensar que es igualmente importante para las diferentes naciones participantes del estudio.

De acuerdo al estudio de Mittal y Dorfman (2012), la dimensión de Egalitarianismo tiene la mayor aceptación en Europa nórdica y las culturas de Europa germánica (> 6,0/7), mientras que la puntuación más baja en esta dimensión corresponde al grupo asiático confusionista $(<5,5 / 7)$. La dimensión de empoderamiento tuvo variabilidad significativa en su aprobación a través de los diferentes grupos de culturas. El grupo de Asia confusionista tuvo la media más baja (5.81/7) y el grupo de culturas anglosajo- nas el puntaje más alto (6.33/7). El grupo de naciones del sur de Asia se ubicó cerca de la mitad del espectro.

La dimensión de la empatía también tuvo variaciones significativas entre culturas. Las naciones del sur de Asia tuvieron la media más alta (5.13/7), y la misma difirió significativamente con la mayoría de los grupos de naciones europeas. El análisis de la varianza de la dimensión de humildad también mostró variaciones significativas en su endoso entre culturas. En las comparaciones entre pares de grupos de países, los países de Europa nórdica, que tuvieron la media más baja $(4,03 / 7)$, difirieron significativamente con los grupos de naciones de Asia meridional, América Latina, Asia confusionista y el grupo de naciones anglosajonas. Las naciones del sur de Asia, con la media más alta $(5,15 / 7)$, difirieron significativamente con los países de Europa nórdica y los de Europa germánica.

Los hallazgos de los estudios empíricos brindan evidencia de que los componentes más importantes del Liderazgo de Servicio son valorados como importantes para el liderazgo efectivo en todas las sociedades. De acuerdo al estudio de Mittal y Dorfman (2012), si bien las diferentes culturas varían en el grado de su aprobación de cuatro dimensiones del liderazgo de servicio, la dimensión de integridad moral es igualmente aprobada como importante en todas las cultu- 
ras. Cabe destacar de este estudio que todas las dimensiones de este estilo de liderazgo fueron evaluadas por encima del punto medio de la escala por todos los grupos de culturas, y las dimensiones de empoderamiento e integridad moral tuvieron medias en todos los grupos de culturas mayores a 6/7.

\section{ANÁLISIS DEL POSIBLE FUNCIONAMIENTO DEL LIDERAZGO DE SERVICIO EN EL CONTEXTO ECUATORIANO}

Tomando como base la revisión de literatura desarrollada en este trabajo sobre la posible aplicación del Liderazgo de Servicio en contextos nacionales con dimensiones culturales específicas, en esta sección se realiza un análisis de la aplicación de este estilo de liderazgo en el contexto ecuatoriano tomando en cuenta los niveles de sus dimensiones culturales. Antes de continuar, es importante mencionar que la literatura existente sobre Liderazgo de Servicio no contiene material específico sobre estudios empíricos que demuestran esa aplicación al contexto ecuatoriano dependiendo de sus dimensiones o características culturales. El presente análisis se basará en estudios teóricos y empíricos sobre la aplicación del Liderazgo de Servicio en culturas con niveles específicos en sus dimensiones, así como también con características específicas de acuerdo al estudio de Mittal y Dorfman (2012).

El Liderazgo de Servicio defiende un rol humanista del líder que no ocupa una posición jerárquica de poder para lograr objetivos, sino que su principal meta es el desarrollo de otros individuos. Consecuentemente, los principales autores que han analizado teóricamente la aplicación de este liderazgo en contextos culturales, por ejemplo Hannay (2009), Mittel y Dorfman (2012), lo han hecho defendiendo la premisa de que el mismo sería mejormente aplicado en culturas con una baja distancia del poder, bajo nivel de individualismo, bajo nivel de masculinidad (equivalente a alta preocupación por la calidad de vida), bajo nivel de evitación de la incertidumbre y una alta orientación al futuro o al largo plazo. Si aplicamos este planteamiento teórico al caso ecuatoriano, los niveles de las dimensiones culturales en este país, en su mayoría no facilitarían la práctica del Liderazgo de Servicio, a excepción del bajo nivel en la dimensión de individualismo.

No obstante, como se mostró en la revisión bibliográfica del presente 
trabajo, existen algunos estudios que evidencian la posibilidad de aplicación del liderazgo de servicio en contextos en los que teóricamente sería muy difícil hacerlo. Por ejemplo, Sousa y Van Dierendonck (2014), encontraron que en una cultura como la portuguesa, con un nivel alto en distancia del poder (63 puntos), se valora la práctica del liderazgo de servicio. De la misma manera, un estudio realizado por Hale y Fields (2007) concluyó que aunque el Liderazgo de Servicio sería de mayor aceptación y aplicación en Estados Unidos que en Ghana, este no es rechazado por la cultura africana, que tiene un alto nivel de distancia del poder, alto nivel de evitación a la incertidumbre y orientación a corto plazo, al igual que el caso ecuatoriano. Finalmente, en un estudio reciente realizado por Cabezas (2016) con niños trabajadores de la calle y sus familias en Ecuador, se pudo identificar la valoración y práctica de este estilo de liderazgo, mismo que produjo cambios positivos significativos en la vida de estos individuos, quienes se convirtieron en líderes de servicio que afectaron positivamente en la vida de otras personas.

En un tipo de análisis diferente, teniendo como referencia los resultados del estudio de Mittal y Dorfman (2012), se han identificado las principales características del liderazgo de servicio (Egalitarianismo, Integridad, Moral, Empoderamiento, Empatía, y Humildad) y se comparan las puntuaciones promedio de las mismas entre América Latina con los promedios globales generales para ubicar el nivel de las mismas en un contexto general. A continuación, se presentan en la tabla 1 los datos de esa comparación.

Tabla 1: Comparación de los promedios de los países de América Latina en las características del Liderazgo de Servicio con los promedios globales. Fuente: Mittal y Dorfman (2012).

\begin{tabular}{|l|c|c|}
\hline Dimensiones de Liderazgo de Servicio & Promedio Global & Promedio de América Latina \\
\hline Egalitarianismo & 5,73 & 5,90 \\
\hline Integridad Moral & 6,12 & 6,35 \\
\hline Empoderamiento & 6,08 & 6,20 \\
\hline Empatía & 4,64 & 4,70 \\
\hline Humildad & 4,62 & 5,00 \\
\hline
\end{tabular}

De los datos de la tabla 1 se puede apreciar que de acuerdo a la medi- ción de Mittal y Dorfman (2012), el grupo de países de América Latina presenta 
promedios superiores a la media global en todas las características principales de Liderazgo de Servicio. Esta información nos sugiere que los países de este grupo, al que pertenece el Ecuador, presentan una congruencia en sus características con los elementos más importantes del liderazgo de servicio en relación con el promedio global de las mismas. Este úl- timo análisis nos muestra evidencias diferentes sobre la posible congruencia de este tipo de liderazgo en culturas como la ecuatoriana. Estos últimos datos sugieren que las características del Liderazgo de Servicio de cierta forma son compatibles con los valores culturales que se comparten en la región a la que el Ecuador pertenece.

\section{DISCUSIÓN DE RESULTADOS Y CONCLUSIONES}

El Liderazgo de Servicio ha sido ampliamente estudiado en los últimos años como una alternativa a los estilos de liderazgo tradicionales que se enfocan principalmente en el logro de objetivos organizacionales. Este tipo de liderazgo defiende la idea de que la motivación principal para liderar en diferentes contextos debe ser el servicio y no el deseo de poder. Hace algunos años ha nacido la inquietud sobre la posible identificación de las variables que afectarían la práctica de este estilo de liderazgo. Entre esas variables, algunos autores se han enfocado en los niveles de dimensiones culturales nacionales. Sobre esta temática se han desarrollado mayormente estudios teóricos que han defendido la hipótesis del mejor funcionamiento de este estilo de liderazgo en culturas nacionales cuyas dimensiones culturales estén relaciona- das con la promoción de la equidad y la calidad de vida. No obstante, varios estudios empíricos han mostrado que este tipo de liderazgo también puede ser aplicable en culturas en los que los niveles de las dimensiones culturales no encajan mayormente con esta premisa. También en los últimos años se han realizado estudios, como el de Mittal y Dorfman (2012), que no se han enfocado en congruencias de las dimensiones culturales con definiciones conceptuales de este tipo de liderazgo, sino en la congruencia directa de las características del mismo con valores culturales nacionales.

El presente estudio, fundamentado en la revisión de literatura existente de estudios teóricos y empíricos, ha encontrado que el liderazgo de servicio puede ser aplicado en diversidad de culturas independientemente de los niveles 
de las diferentes dimensiones culturales. La flexibilidad de su aplicación podría ser resultado principalmente la interacción de la práctica de este tipo de liderazgo con otras variables del contexto y el sentido dinámico del entendimiento y la práctica misma del liderazgo independientemente del contexto nacional.

Sobre esta misma posibilidad, Pekerti y Sendjaya (2010), basándose en una revisión del estudio GLOBE de 62 sociedades (House et al., 2004) adoptaron la teoría del "liderazgo implícito" para afirmar que el concepto de liderazgo puede encapsular una diversidad de significados y diferentes puntos de referencia. Por esa razón, el mismo Liderazgo de Servicio podría ser interpretado de diferente manera, y con diferentes especificidades dependiendo del contexto. La teoría del liderazgo implícito reconoce al liderazgo como un concepto socialmente construido que es filtrado, interpretado y actuado de maneras muy diferentes, dependiendo de diversas perspectivas cognitivas y circunstancias experienciales (Bresnan 1995 Gerstner y Day 1994; Ashkanasy 2004; Holmberg y Akerblom 2006). Si el liderazgo es un fenómeno socialmente construido, entonces hay diferencias inevitables en lo que se entiende como liderazgo en diferentes culturas (Abdalla y Al-Homoud 2001; Dastmalchian, Javidan y Alam 2001; Ling, Chia y
Fang 2000). Al mismo tiempo, también se espera que existan algunas similitudes en lo que constituye el liderazgo entre culturas basado en la importancia y las prioridades de las metas, incluyendo lo que las partes interesadas consideran necesario (Bass 1990; Dorfman, Hanges y Brodbeck. 2004; Westwood y Posner 1997).

Las investigaciones sobre el tema del liderazgo han brindado cada cierto tiempo nuevas propuestas de estilos eficaces dependiendo de los contextos. Los estilos de liderazgo varían en sus características, pero se puede apreciar que existen dimensiones transversales que se repiten en los estilos contemporáneos de liderazgo, que en la actualidad afrontan crisis de corrupción en nuestras sociedades a nivel global. Sobre este aspecto, Shekari, Taft, Yazd y Nikooparvar Mahmood (2012) afirmaron: "el mundo clama por un liderazgo ético y eficaz de servicio a los demás. Invertir en su desarrollo y cumplir una visión compartida "(pág.1). Por estas razones, el componente ético del Liderazgo de Servicio es de aplicación y necesidad global.

Los resultados del presente trabajo de recopilación bibliográfica apuntan a sugerir que este tipo de liderazgo puede ser aplicado con éxito en diferentes culturas nacionales alrededor del mundo, incluyendo la ecuatoriana. Existirán algu- 
nas variables, como los niveles de dimensiones culturales, que de cierta manera influencien la percepción de este tipo de liderazgo y de alguna forma su acepta- ción y funcionamiento. No obstante, su práctica es bienvenida e inminentemente necesaria.

\section{RECOMENDACIONES PARA FUTURAS INVESTIGACIONES SOBRE EL TEMA}

La proliferación de estudios sobre el Liderazgo de Servicio realizados en las últimas décadas, en los que se han mostrado sus principales características y sus beneficios, han permitido que se genere una gran aceptación del mismo por parte de gestores del desarrollo organizacional. Uno de los temas que ha generado más interés sobre este tipo de liderazgo es la identificación de las variables que afectarían su aceptación y práctica. En el presente trabajo, que ha analizado la posible aplicabilidad de este tipo de liderazgo de- pendiendo de los niveles de las dimensiones culturales de una nación específica, y sus características. Se ha concluido que la incongruencia de esas dimensiones y características podría afectar de cierta forma su funcionamiento. No obstante, el mismo no se ve impedido. Son necesarios más estudios empíricos que incorporen nociones como la del liderazgo implícito para controlar variables específicas como el mismo entendimiento del Liderazgo de Servicio dentro de los contextos nacionales diferentes. 


\section{BIBLIOGRAFÍA}

Abdalla, I.A., y Al-Homoud, A.M. (2001), Exploring the Implicit Leadership Theory in the Arabian Gulf States, Applied Psychology: An International Review, 50, 506-531.

Ashkanasy, N.M. (2004), Leadership in the Asian Century: Lessons from GLO$\mathrm{BE}$, International Journal of Organisational Behaviour, 5, 150-163.

Barbuto, J. E., y Wheeler, D. (2006). Scale Development and Construct Clarification of Servant Leadership. Group y Organization Management, 31(3): 300-326

Barbuto, J. E., Gottfredson, R. K., y Searle, T. P. (2014). An examination of emotional intelligence as an antecedent of servant leadership. Journal of Leadership y Organizational Studies. 21, (3), 315.

Bass, B. M. (1990), Bass y Stogdill's Handbook of Leadership: Theory, Research and Managerial Applications (3rd ed.), New York: Free Press.

Beazley, D., y Gemmill, G. (2006). Spirituality and Servant Leader Behavior. Journal of Management, Spirituality y Religion, 3(3), 258-270.

Bresnan, W.J. (1995), All Things to all People? Perceptions, Attributions, and Constructions of Leadership,
Leadership Quarterly, 6, 495-513. Cabezas, C. B. (2016). El Liderazgo de Servicio y su efecto transformador: el caso del Centro del Muchacho Trabajador. Revista de la Pontificia Universidad Católica del Ecuador. 103, 3-31.

Carroll, B. C., y Patterson, K. (2014). Servant leadership: A cross cultural study between India and the United States. Servant Leadership: Theory y Practice, 1(1), 16-45.

Cattell, R. B. (1949). The dimensions of culture patterns by factorization of national characters. The Journal of Abnormal and Social Psychology, 44(4), 443.

Chanchani, S., y Theivananthampillai, P. (2009). Typologies of culture. Available at SSRN 1441609.

Choi, Y., y Yoon, J. (2005), Effects of Leaders' Self-sacrificial Behavior and Competency on Followers Attribution of Charismatic Leadership among Americans and Koreans, Current Research in Social Psychology, 11, 51-69.

Dastmalchian, A., Javidan, M., y Alam, K. (2001), Effective Leadership and Culture in Iran: An Empirical Study, Applied Psychology: An International Review, 50, 532-558. 
Dennis, R. S., y Bocarnea, M. (2005). Development of the servant leadership assessment instrument. Leadership and Organization Development Journal, 25(8), 600-615.

Dorfman, P. W., Hanges, P. J., y Brodbeck, F. C. (2004). Leadership and cultural variation: The identification of culturally endorsed leadership profiles. En R. J. House, P. J. Hanges, M. Javidan, P. W. Dorfman, y V. Gupta (Eds.), Culture, leadership, and organizations: The GLOBE study of 62 societies (pp. 669-720). Thousand Oaks, CA: Sage.

Gerstner, C.R., y Day, D.V. (1994), 'Cross-cultural Comparison of Leadership Prototype, Leadership Quarterly, 5, 121-134.

Greenleaf, R. K. (1970). Servant leadership: A journey into the nature of legitimate power and greatness. New York: Paulist Press.

Greenleaf, R. K. (1977). Servant leadership: A journey into the nature of legitimate power and greatness. New York: Paulist Press.

Hale, J. R., y Fields, D. L. (2007). Exploring servant leadership across cultures: A study of followers in Ghana and the USA. Leadership, 3(4), 397417.

Han, Y., Kakabadse, N. K., y Kakabadse, A. (2010). Servant leadership in the People's Republic of China: A case study of the public sector. Journal of Management Development, 29(3), 265-281.

Hannay, M. (2009). The cross-cultural leader: The application of servant leadership theory in the international context. Journal of International Business and Cultural Studies. Recuperado de: http://www. aabri.com/manuscripts/08108. pdf

Harwiki, W. (2016). The Impact of Servant Leadership on Organization Culture, Organizational Commitment, Organizational Citizenship Behaviour (OCB) and Employee Performance in Women Cooperatives. Procedia-Social and Behavioral Sciences, 219, 283-290.

Hofstede, G. (1980). Culture's Consequences: International Differences in Work-Related Values. Beverly Hills, CA: Sage.

Hofstede, G., y Bond, M. H. (1988). The Confucius connection: From cultural roots to economic growth. Organizational dynamics, 16(4), 5-21.

Hofstede, G. (2017). Geert Hofstede ${ }^{\mathrm{TM}}$ Ecuador: Cultural Dimensions. Recuperado el 11 de mayo de 2017 de: http://www.geert-hofstede. com/hofstede_ecuador.shtml

Holmberg, I., y A kerblom, S. (2006). Modelling leadership_Implicit lea- 
dership theories in Sweden. Scandinavian Journal of Management, 22(4): 307-329.

House, R., Hanges, P., Javidan, M., Dorman, P., y Gupta, V. (Eds.). (2004). Culture, leadership, and organizations: The GLOBE study of 62 societies. Thousand Oaks, CA: Sage.

House, R., Javidan, M., Hanges, P., y Dorfman, P. (2002). Understanding cultures and implicit leadership theories across the globe: an introduction to project GLOBE. Journal of world business, 37(1), 3-10.

Inkeles, A., y Levinson, D. J. (1969). National character: The study of modal personality and sociocultural systems. The handbook of social psychology, 4, 418-506.

Javidan, M., House, R. J., y Dorfman, P. W. (2004). A nontechnical summary of GLOBE findings. En R. J. House, P. J. Hanges, M. Javidan, P. W. Dorfman, y V. Gupta (Eds.), Culture, leadership, and organizations: The GLOBE Study of 62 societies (pp. 29-48). Thousand Oaks, CA: Sage.

Koesmono, H. T. (2014). The Influence of Organizational Culture, Servant Leadership, and Job Satisfaction Toward Organizational Commitment and Job Performance Through Work Motivation as Mo- derating Variables for Lecturers in Economics and Management of Private Universities in East Surabaya. Educational Research International, 3(4).

Kroeber, A. L., y Kluckhohn, C. (1952). Culture: A critical review of concepts and definitions. Papers. Peabody Museum of Archaeology y Ethnology, Harvard University.

Laub, J. (1999). Assessing the Servant Organization: Development of the Servant Organizational Leadership Assessment (SOLA) Instrument. Unpublished PhD Dissertation, Florida Atlantic University

Liden, R. C., Wayne, S. J., Zhao, H., y Henderson, D. (2008). Servant leadership: Development of a multidimensional measure and multi-level assessment. The leadership quarterly, 19(2), 161-177.

Ling, W., Chia, R.C., y Fang, L. (2000), Chinese Implicit Leadership Theory, The Journal of Social Psychology, 140, 729-739.

Machado, D. y Carvalho, C.E. (2008) Cultural Typologies and Organizational Environment: A Conceptual Analysis, Latin American Business Review, 9(1), 1-32.

Mittal, R., y Dorfman, P.W. (2012). Servant leadership across cultures. Journal of World Business, 47(4), 555570. 
Molero, A. (2002). Cultura y liderazgo. Una relación multifacética. Boletín de Psicología, (76), 53-76.

Northouse, P. (2013). Leadership: Theory and practice (6th ed.). Thousand Oaks, CA: Sage.

Page, D., y P. Wong. (2000). A Conceptual Framework for Measuring Servant Leadership. En The Human Factor in Shaping the Course of History and Development, ed., S. Adjibolosoo, Lanham, MD: University Press of America.

Parris, D. L., y Peachey, J. W. (2013). A systematic literature review of servant leadership theory in organizational contexts. Journal of business ethics, 113(3), 377-393.

Pekerti, A. A., y Sendjaya, S. (2010). Exploring servant leadership across cultures: comparative study in Australia and Indonesia. The International Journal of Human Resource Management, 21(5), 754-780. https://doi.org /10.1080/09585191003658920

Reed, L. L., Vidaver-Cohen, D., y Colwell, S. R. (2011). A new scale to measure executive servant leadership: Development, analysis, and implications for research. Journal of Business Ethics, 101(3), 415-434.

Rubio-Sánchez, A., Bosco, S. M., y Melchar, D. E. (2013). Servant Leadership and World Values. Global
Studies Journal, 5(3), 19-33.

Rummel, R. J. (1972). The dimensions of nations (Vol. 1). Beverly Hills, CA: Sage Publications

Russell, R. F., y Stone, A. G.. 2002. A Review of Servant Leadership Attributes: Developing a Practical Model. Leadership y Organization Development Journal, 23(3/4), 145-158.

Russell, R.F. (2001). The role of values in servant leadership. Leadership y Organization Development Journal, 22(2), 76-83.

Sawyer, J. (1967). Dimensions of nations: Size, wealth, and politics. American Journal of Sociology, 73, 145172.

Shekari, H., Taft, Yazd, I. R., \& Nikooparvar Mahmood, Z. (2012). Promoting leadership effectiveness in organizations: A case study on the involved factors of servant leadership. International Journal of Business Administration, 3(1), 54-65.

Shi, X., y Wang, J. (2011). Interpreting Hofstede model and Globe model: which way to go for cross-cultural research?. International journal of business and management, 6(5), 93.

Smith, B. N., Montagno, R. V., y Kuzmenko, T. N. (2004). Transformational and Servant Leadership: Content and Contextual Comparisons. Journal of Leadership y Organizational Stu- 
dies, 10, 80-91

Song, C., Park, K. R., y Kang, S. W. (2015). Servant leadership and team performance: The mediating role of knowledge-sharing climate. Social Behavior and Personality: an international journal, 43(10), 17491760.

Sousa M., y van Dierendonck D. (2014). Servant leadership and engagement in a merge process under high uncertainty. Change Management. 27, 877-899.

Spears, L. C. (2005). The understanding and practice of servant leadership. International Journal of Servant Leadership, 1(1), 29-46.

Spears, L.C. (Ed.). (1998). Insights on leadership: Service, stewardship, spirit and servant-leadership. New York, NY: John Wiley y Sons.

Sun, P. Y. T. (2013). The servant identity: Influences on the cognition and behavior of servant leaders. Leadership Quarterly, 24, 544-557.

Top, S., Öge, E., Atan, Ö., y Gümüş, S. (2015). Investigation Relational Levels of Intensity between Paternalistic and Servant Leadership Styles and National Culture, Organizational Commitment and Subordinate Responses or Reactions to the Leaders Style. Procedia-Social and Behavioral Sciences, 181, 12-22.
Triandis, H. (1994). Culture and Social Behavior. New York: McGraw-Hill

Trompenaars, F. y Hampden-Turner, C. (1997), Riding the Waves of Culture: Understanding Cultural Diversity in Business, Second Edition. London y Santa Rosa: Nicholas Brealey Publising Limited

Tulasi Das, V., y Anand Reddy, S. (2013). Influence Of Personality Factors On Servant Leadership: An Empirical Study. Journal on Management, 8(1).

Van Dierendonck, D. (2010). Servant Leadership: A Review and Synthesis. Journal of Management, 37(4), 1228-1261. http://doi. org/ 10.1177/0149206310380462

Van Dierendonck, D. (2011). Servant leadership: A review and synthesis. Journal of Management, 37, 12281261.

Van Dierendonck, D., y Nuijten, I. (2011). The servant leadership survey: Development and validation of a multidimensional measure. Journal of business and psychology, 26(3), 249-267.

Verdorfer, A. P. (2016). Examining Mindfulness and Its Relations to $\mathrm{Hu}$ mility, Motivation to Lead, and Actual Servant Leadership Behaviors. Mindfulness, 7, (4), 1-12.

Washington, R. R., Sutton, C. D., y Feild, H. S. (2006). Individual differences 
in servant leadership: The roles of values and personality. Leadership y Organization Development Journal, 27(8), 700-716.

Westwood, R.I., y Posner, B.Z. (1997). Managerial Values across Cultures:
Australia, Hong Kong and the United States, Asia Pacific Journal of Management, 14, 31-66.

World Values Association. (2013). World Values Survey. World Values Research. 